International Journal of Applied Mathematics

Volume 33 No. $5 \quad 2020,867-877$

ISSN: $1311-1728$ (printed version); ISSN: 1314-8060 (on-line version)

doi: http://dx.doi.org/10.12732/ijam.v33i5.9

\title{
STUDY OF ERROR OF APPROXIMATION OF CONJUGATE FOURIER SERIES IN WEIGHTED \\ CLASS BY ALMOST RIESZ MEANS
}

\author{
Kusum Sharma \\ Department of Mathematics \\ National Institute of Technology \\ Uttarakhand - 246174, INDIA
}

\begin{abstract}
The present work is aimed to study error (or degree) of approximation of a function $\tilde{g}$, conjugate to $2 \pi$-periodic function $g$, belonging to weighted $W\left(L^{p}, \xi(t)\right)(p \geq 1)$ by almost Riesz means. Our result gives sharper estimates than the previous known results. Some of important corollaries have also been derived from our main theorem.
\end{abstract}

AMS Subject Classification: 40C99, 40G99, 41A25, 42A16

Key Words: degree of approximation; almost Riesz means; $W\left(L^{p}, \xi(t)\right)$ $(p \geq 1)$-class; Fourier series

\section{Introduction}

Let $g \in L^{p}(p \geq 1)$ be a $2 \pi$-periodic and Lebesgue integrable function. Then the Fourier series of $g(x)$ at any point $x$ is given by

$$
g \sim \frac{a_{0}}{2}+\sum_{n=1}^{\infty}\left(a_{n} \cos n x+b_{n} \sin n x\right)
$$

with $s_{n}(g ; x)$, i.e. $n^{\text {th }}$ partial sum which is also called trigonometric polynomial of $n^{\text {th }}$ order (or degree) of Fourier series.

Received: July 1, 2020

(C) 2020 Academic Publications 
The conjugate series of (1) is given by

$$
\sum_{n=1}^{\infty}\left(b_{n} \cos n x-a_{n} \sin n x\right),
$$

where the conjugate function $\tilde{g}$ is defined as

$$
\tilde{g}(x)=-\frac{1}{2 \pi} \int_{0}^{2 \pi} \psi(t) \cot \frac{t}{2} d t .
$$

A function $g$ is said to belong to Lip $\alpha$ class if

$$
|g(x+t)-g(x)|=O\left(|t|^{\alpha}\right) \text { for } 0<\alpha \leq 1 \text {. }
$$

A function $g \in \operatorname{Lip}(\alpha, p)(p \geq 1)$-class for $a \leq x \leq b$, if

$$
\left\{\int_{a}^{b}|g(x+t)-g(x)|^{p} d x\right\}^{\frac{1}{p}} \leq K\left(|t|^{\alpha}\right), \quad 0<\alpha \leq 1
$$

where $K$ is absolute non-negative constant (Definition 5.38 of McFadden [12]).

If $g \in \operatorname{Lip}(\xi(t), p)(p \geq 1)$-class, then

$$
\left\{\int_{0}^{2 \pi}|g(x+t)-g(x)|^{p} d x\right\}^{\frac{1}{p}}=O(\xi(t)), \quad 0 \leq x \leq 2 \pi, t>0 .
$$

A function $g \in W\left(L^{p}, \xi(t)\right)(p \geq 1)$-class [5], if

$$
\left\{\int_{0}^{2 \pi}|g(x+t)-g(x)|^{p} \sin ^{\beta p}\left(\frac{x}{2}\right) d x\right\}^{\frac{1}{p}}=O(\xi(t)), \beta \geq 0, t>0 .
$$

Here $\xi(t)$ is non-negative increasing function of $t$. The weighted Lipschitz $W\left(L^{p}, \xi(t)\right)(p \geq 1)$-class reduces to $\operatorname{Lip}(\xi(t), p)$ class if we take $\beta=0$. If we put $\xi(t)=t^{\alpha}$, then $\operatorname{Lip}(\xi(t), p)$ class is converted to $\operatorname{Lip}(\alpha, p)$, and if $p \rightarrow \infty$ in $\operatorname{Lip}(\alpha, p)$ class, then it reduces to Lip $\alpha$. Thus $W\left(L^{p}, \xi(t)\right)$ class is the generalized Lipschitz class.

The $L_{\infty}$-norm of a function $g$ is defined as $\|g\|_{\infty}=\sup \{|g|: x \in R\}$ and the $L_{p}$-norm of a function $g: R \rightarrow R$ is defined as

$$
\|g\|_{p}=\left(\int_{0}^{2 \pi}|g(x)|^{p} d x\right)^{\frac{1}{p}}, 1 \leq p<\infty .
$$

The error approximation of a function $g: R \rightarrow R$ is defined by

$$
E_{n}(g)=\left\|s_{n}(x)-g(x)\right\|_{\infty}=\sup _{x \in R}\left\{\left|s_{n}(x)-g(x)\right|\right\},
$$


where $E_{n}(g)$ is defined under sup norm $\|\cdot\|_{\infty}[1]$. If $g \in L^{p}$, then $E_{n}(g)=$ $\min _{x}\left\|s_{n}-g\right\|_{p}$.

The above method of approximation is called a trigonometric Fourier approximation (TFA).

If $g(x)$ and $\phi(x)$ both are integrable in the range $a \leq x \leq b$ and $\phi(x)$ is monotonic in the same range, i.e. for $x \in[a, b]$. Then $\exists \lambda \in(a, b)$ such that

$$
\int_{a}^{b} g(x) \phi(x) d x=\phi(a) \int_{a}^{\lambda} g(x) d x+\phi(b) \int_{\lambda}^{b} g(x) d x .
$$

The above is known as the second mean value theorem for integrals.

Let $\sum_{n=0}^{\infty} x_{n}$ be an infinite series with the sequence of its $n^{\text {th }}$ partial sum $\left\{s_{n}\right\}$. Now we give some basic definitions which will be useful in this paper.

Definition 1.1. (Lorentz [3]). A sequence $\left\{s_{n}\right\}$ is said to be almost convergent to a finite number $s$ if

$$
\lim _{n \rightarrow \infty} \frac{1}{n+1} \sum_{\mu=p}^{n+p} s_{\mu}=s \text { uniformly with respect to } p .
$$

An almost convergence is the generalization of ordinary convergence. It can be easily seen that a convergent sequence is almost convergent but vise-versa is not always true.

Definition 1.2. (Sharma and Qureshi [16]). Let $\left\{p_{n}\right\}$ be a sequence of positive constant such that $p_{0}>0$, and

$$
P_{n}=p_{0}+p_{1}+p_{2}+\ldots \ldots+p_{n}
$$

Then the conjugate Fourier series is said to be almost Riesz summable to $s$, provided

$$
\tilde{\tau}_{n, p}=\frac{1}{P_{n}} \sum_{k=0}^{n} p_{k} \tilde{s}_{k, p} \rightarrow s, \text { as } n \rightarrow \infty
$$

uniformly with respect to $p$, where

$$
\tilde{s}_{k, p}=\frac{1}{k+1} \sum_{\mu=1}^{k+1} \tilde{s}_{\mu} .
$$

It should be noted that here $\tilde{\tau}_{n, p}$ is also trigonometric polynomial.

The Riesz means is regular if and only if $P_{n} \rightarrow \infty$ (Theorem 1.4.4 of Peterson [4]).

Definition 1.3. For $p \geq 1$ and $\frac{1}{p}+\frac{1}{q}=1$, the Hölder inequality for integrals is given by

$$
\|f g\|_{1}=\|f\|_{p}\|g\|_{q}
$$


where $f$ and $g$ are continuous functions on $[a, b]$. Here $\|f\|_{p}$ is defined as

$$
\|f\|_{p}=\left(\int_{a}^{b}|f(x)|^{p} d x\right)^{1 / p}, 1 \leq p<\infty .
$$

We shall use following notations throughout this paper.

$$
\begin{aligned}
\psi_{x}(t) & =g(x+t)-g(x-t) \\
\tilde{G}_{n}(t) & =\frac{1}{2 \pi P_{n}} \sum_{k=0}^{n} \frac{p_{k}}{k+1} \frac{\cos (k+2 p+1) \frac{t}{2} \sin (k+1) \frac{t}{2}}{\sin ^{2} \frac{t}{2}} .
\end{aligned}
$$

\section{Main theorem}

The degree of approximation of functions belong to $\operatorname{Lip} \alpha, \operatorname{Lip}(\alpha, p), \operatorname{Lip}(\xi(t), p)$ and $W\left(L^{p}, \xi(t)\right)(p \geq 1)$ - classes using different summability methods has been addressed by several investigators like Nig-am and Sharma [6, 7], Mishra and Rhoades [13], Khan [5], Mittal and Mishra [14], Sharma and Malik [10] and Sharma [11]. A good amount of work has been done on almost convergent sequences by many researchers including King [8], Lorentz [3], Mohapatra [17], Ahmad and Mursaleen [19] and Nanda [18]. Almost convergence led to the foundation of almost summability methods. Qureshi [9] studied degree of approximation of function belonging to Lip $\alpha$ class by almost Riesz means of conjugate Fourier series. Thereafter the work of Qureshi [9] has been generalized by Sharma and Sinha [15] in $\operatorname{Lip}(\xi(t), p)$-class. Working in the same direction, in this paper, we generalize the result obtained by Sharma and Sinha [15] under some set conditions.

We utilize almost Riesz means to get the degree of approximation of conjugate function in weighted $W\left(L^{p}, \xi(t)\right)(p \geq 1)$ space using trigonometric Fourier approximation method. In fact, we prove the following result:

Theorem 2.1. Let $\left\{p_{n}\right\}$ be a monotonic increasing sequence. Then the error of approximation a function $\tilde{g}(x)$, conjugate to a $2 \pi$-periodic, Lebesgue integrable function, belonging to weighted Lipschitz $W\left(L^{p}, \xi(t)\right)(p \geq 1)$-class by almost Riesz means of conjugate series (2) is given by

$$
\left|\tilde{\tau}_{n, p}(x)-\tilde{g}(x)\right|=O\left\{\xi\left(\frac{1}{P_{n}}\right) P_{n}^{\beta+\frac{1}{p}}\right\},
$$

where $\tilde{\tau}_{n, p}(x)$ is the almost Riesz means of the conjugate series (2) and $\xi(t)$ is positive increasing function, satisfies the following conditions:

$$
\frac{\xi(t)}{t} \text { is non-increasing in } t,
$$




$$
\begin{gathered}
{\left[\int_{0}^{\pi / P_{n}}\left(\frac{t\left|\psi_{x}(t)\right| \sin ^{\beta} \frac{t}{2}}{\xi(t)}\right)^{p} d t\right]^{1 / p}=O\left(P_{n}^{-1}\right),} \\
{\left[\int_{\pi / P_{n}}^{\pi}\left(\frac{t^{-\delta}\left|\psi_{x}(t)\right|}{\xi(t)}\right)^{p} d t\right]^{1 / p}=O\left(P_{n}^{\delta}\right),}
\end{gathered}
$$

where $\delta$ is an arbitrary number such that $0<\delta<\beta+\frac{1}{p}$ and $p+q=p q$ for $1 \leq p<\infty$. The conditions (9) and (10) hold uniformly in $x$ and

$$
\tilde{g}(x)=-\frac{1}{2 \pi} \int_{0}^{2 \pi} \psi(t) \cot \frac{t}{2} d t .
$$

\section{Lemma}

In order to prove our theorem, we need following lemma:

Lemma 3.1. $\left|\tilde{G}_{n}(t)\right|=O\left(\frac{1}{t}\right)$ for $0<t \leq \frac{\pi}{P_{n}}$.

Proof. Applying the well known inequalities $\sin n t \leq n \sin t$ and $|\cos t| \leq 1$, we have

$$
\begin{aligned}
\left|\tilde{G}_{n}(t)\right| & =\frac{1}{2 \pi P_{n}}\left|\sum_{k=0}^{n} \frac{p_{k}}{k+1} \frac{\cos (k+2 p+1) \frac{t}{2} \sin (k+1) \frac{t}{2}}{\sin ^{2} \frac{t}{2}}\right| \\
& =\frac{1}{2 \pi P_{n}} \sum_{k=0}^{n} \frac{p_{k}}{k+1} \frac{(k+1)}{t / \pi} \\
& =\frac{1}{2 t P_{n}} \sum_{k=0}^{n} p_{k} \\
& =O\left(\frac{1}{t}\right), \quad \text { since } \sum_{k=0}^{n} p_{k}=P_{n} .
\end{aligned}
$$

The proof is completed. 


\section{Proof of Theorem}

The integral representation of partial sums $\tilde{s}_{k}(x)$ of the conjugate Fourier series (2) is given by

and

$$
\tilde{s}_{k}(x)-\tilde{g}(x)=-\frac{1}{\pi} \int_{0}^{\pi} \psi_{x}(t) \frac{\cos \left(k+\frac{1}{2}\right) t}{2 \sin \frac{t}{2}} d t
$$

$$
\begin{aligned}
\tilde{s}_{k, p}(x)-\tilde{g}(x) & =\frac{1}{k+1} \sum_{\mu=k}^{k+p}\left(\tilde{s}_{\mu}(x)-\tilde{g}(x)\right) \\
& =-\frac{1}{\pi(k+1)} \int_{0}^{\pi} \psi_{x}(t) \sum_{\mu=k}^{k+p} \frac{\cos \left(\mu+\frac{1}{2}\right) t}{2 \sin \frac{t}{2}} d t \\
& =\frac{1}{2 \pi(k+1)} \int_{0}^{\pi} \psi_{x}(t) \frac{\sin p t-\sin (k+p+1)}{2 \sin ^{2} \frac{t}{2}} d t .
\end{aligned}
$$

Representing the almost Riesz means of partial sum $\tilde{s}_{k, p}(x)$ by $\tilde{\tau}_{n, p}(x)$, we can write

$$
\begin{aligned}
\tilde{\tau}_{n, p}(x)-\tilde{g}(x) & =\frac{1}{P_{n}} \sum_{k=0}^{n}\left(\tilde{s}_{k, p}(x)-\tilde{g}(x)\right) \\
& =\frac{1}{2 \pi P_{n}} \int_{0}^{\pi} \psi_{x}(t) \sum_{k=0}^{n} \frac{p_{k}}{k+1} \frac{\sin p t-\sin (k+p+1)}{2 \sin ^{2} \frac{t}{2}} d t .
\end{aligned}
$$

Therefore,

$$
\begin{aligned}
\left|\tilde{\tau}_{n, p}(x)-\tilde{g}(x)\right| & \leq \int_{0}^{\pi}\left|\psi_{x}(t)\right|\left|\tilde{G}_{n}(t)\right| d t \\
& =\int_{0}^{\frac{\pi}{P_{n}}}\left|\psi_{x}(t)\right|\left|\tilde{G}_{n}(t)\right| d t+\int_{\frac{\pi}{P_{n}}}^{\pi}\left|\psi_{x}(t)\right|\left|\tilde{G}_{n}(t)\right| d t \\
& =I_{1}+I_{2} \quad \text { (say). }
\end{aligned}
$$

Now considering

$$
I_{1}=\int_{0}^{\frac{\pi}{P_{n}}}\left(\left|\psi_{x}(t)\right|\left|\tilde{G}_{n}(t)\right|\right) d t
$$

and using Lemma 3.1, we have

$$
I_{1}=\int_{0}^{\frac{\pi}{P_{n}}}\left(\frac{\left|\psi_{x}(t)\right|}{t}\right) d t
$$


Since $\tilde{g} \in W\left(L^{p}, \xi(t)\right) \Rightarrow \psi_{x}(t) \in W\left(L^{p}, \xi(t)\right)$-class, using the Hölder inequality and condition (9), we get

$$
\begin{aligned}
I_{1} & =\left(\int_{0}^{\frac{\pi}{P_{n}}}\left(\frac{t\left|\psi_{x}(t)\right| \sin ^{\beta} t / 2}{\xi(t)}\right)^{p} d t\right)^{\frac{1}{p}}\left(\int_{0}^{\frac{\pi}{P_{n}}}\left(\frac{\xi(t)}{t^{2} \sin ^{\beta} t / 2}\right)^{q} d t\right)^{\frac{1}{q}} \\
& =O\left(P_{n}^{-1}\right)\left(\int_{0}^{\frac{\pi}{P_{n}}}\left(\frac{\xi(t)}{t^{2} \sin ^{\beta} t / 2}\right)^{q} d t\right)^{\frac{1}{q}} .
\end{aligned}
$$

Using the second mean value theorem for integrals and the inequality $(\sin t / 2) \leq$ $\frac{\pi}{t}$ for $0 \leq t \leq \pi$, we have

$$
\begin{aligned}
I_{1} & =O\left(P_{n}^{-1} \xi\left(\frac{1}{P_{n}}\right)\right)\left(\lim _{\epsilon \rightarrow 0} \int_{\epsilon}^{\frac{\pi}{P_{n}}}\left(\frac{1}{t^{\beta+2}}\right)^{q} d t\right)^{\frac{1}{q}} \\
& =\left(P_{n}^{-1} \xi\left(\frac{1}{P_{n}}\right)\right)\left(\frac{t^{-(\beta+2)+\frac{1}{q}}}{-(\beta+2)+\frac{1}{q}}\right)_{\epsilon}^{\frac{\pi}{P_{n}}} \\
& =\left(P_{n}^{-1} \xi\left(\frac{1}{P_{n}}\right) \cdot P_{n}^{\beta+1+\left(1-\frac{1}{q}\right)}\right) \\
& =O\left(\xi\left(\frac{1}{P_{n}}\right) P_{n}^{\beta+\frac{1}{p}}\right), \quad \text { since } \frac{1}{p}+\frac{1}{q}=1 .
\end{aligned}
$$

Consider

$$
\begin{aligned}
I_{2} & =\int_{\frac{\pi}{P_{n}}}^{\pi}\left|\psi_{x}(t)\right|\left|\tilde{G}_{n}(t)\right| d t \\
& =\frac{1}{2 \pi P_{n}} \int_{\frac{\pi}{P_{n}}}^{\pi}\left(\left|\psi_{x}(t)\right| \sum_{k=0}^{n} \frac{p_{k}}{k+1} \frac{\cos (k+2 p+1) \frac{t}{2} \cdot \sin (k+1) \frac{t}{2}}{\sin ^{2} \frac{t}{2}}\right) d t .
\end{aligned}
$$

Again using the Hölder inequality, we have

$$
\begin{aligned}
I_{2} & =O\left(\frac{1}{P_{n}}\right)\left(\int_{\frac{\pi}{P_{n}}}^{\pi}\left(\frac{t^{-\delta}\left|\psi_{x}(t)\right| \sin ^{\beta} \frac{t}{2}}{\xi(t)}\right)^{p} d t\right)^{\frac{1}{p}} \times \\
& \left(\int_{\frac{\pi}{P_{n}}}^{\pi}\left(\frac{\xi(t)}{t^{-\delta} \sin ^{\beta} \frac{t}{2}} \sum_{k=0}^{n} \frac{p_{k}}{k+1} \frac{\cos (k+2 p+1) \frac{t}{2} \cdot \sin (k+1) \frac{t}{2}}{\sin ^{2} \frac{t}{2}}\right)^{q} d t\right)^{\frac{1}{q}} .
\end{aligned}
$$


Using inequalities $|\sin n t| \leq 1,\left|\sin \frac{t}{2}\right| \leq \frac{\pi}{t}$, and condition (10), we obtain

$$
\begin{aligned}
& I_{2}=O\left(\frac{1}{P_{n}}\right) O\left(P_{n}^{\delta}\right) c \\
& \times\left(\int_{\frac{\pi}{P_{n}}}^{\pi}\left(\frac{\xi(t)}{t^{-\delta} \sin ^{\beta} \frac{t}{2}} \sum_{k=0}^{n} \frac{p_{k}}{k+1} \cdot \frac{(k+1) \cos (k+2 p+1) \frac{t}{2}}{\frac{t}{\pi}}\right)^{q} d t\right)^{\frac{1}{q}} \\
& =O\left(P_{n}^{\delta-1}\right)\left(\int_{\frac{\pi}{P_{n}}}^{\pi}\left(\frac{\xi(t)}{t^{-\delta+\beta+1}} \sum_{k=0}^{n} p_{k} \cos (k+2 p+1) \frac{t}{2}\right)^{q} d t\right)^{\frac{1}{q}} \\
& =O\left(P_{n}^{\delta-1}\right)\left(\int_{\frac{\pi}{P_{n}}}^{\pi}\left(\frac{\xi(t)}{t^{-\delta+\beta+1}}\right)^{q} d t\right)^{\frac{1}{q}} .
\end{aligned}
$$

Since $\left\{p_{n}\right\}$ is monotonic increasing [16], we have

$$
\begin{aligned}
\sum_{k=0}^{n} p_{k} \cos (k+2 p+1) \frac{t}{2} & \leq p_{n} \max _{0 \leq \nu \leq n} \sum_{k=0}^{\nu} \cos (k+2 p+1) \frac{t}{2} \\
& =O\left(\frac{p_{n}}{t}\right) .
\end{aligned}
$$

Putting $t=\frac{1}{y} \Rightarrow d t=-\frac{1}{y^{2}} d y$, we get

$$
I_{2}=O\left(P_{n}^{\delta-1}\right)\left(\int_{\frac{1}{\pi}}^{\frac{P_{n}}{\pi}}\left(\frac{y \xi\left(\frac{1}{y}\right)}{y^{\delta-\beta-1}}\right)^{q} \frac{d y}{y^{2}}\right)^{\frac{1}{q}} .
$$

Since $\frac{\xi(t)}{t}$ is decreasing function $\Rightarrow \frac{\xi\left(\frac{1}{y}\right)}{\frac{1}{y}}=y \xi\left(\frac{1}{y}\right)$ is increasing. Therefore applying second mean value theorem for integrals, we get

$$
\begin{aligned}
& =O\left(P_{n}^{\delta-1}\right)\left(P_{n} \cdot \xi\left(\frac{1}{P_{n}}\right)\right)\left(\int_{\frac{1}{\pi}}^{\frac{P_{n}}{\pi}} y^{(\beta+1-\delta) q-2} d y\right)^{\frac{1}{q}} \\
& =O\left(P_{n}^{\delta} \xi\left(\frac{1}{P_{n}}\right)\right)\left(\frac{y^{(\beta+1-\delta)-\frac{1}{q}}}{(\beta+1-\delta)-\frac{1}{q}}\right)_{\frac{1}{\pi}}^{\frac{P_{n}}{\pi}} \\
& =O\left(P_{n}^{\delta} \xi\left(\frac{1}{P_{n}}\right) P_{n}^{(\beta+1-\delta)-\frac{1}{q}}\right) \\
& =O\left(\xi\left(\frac{1}{P_{n}}\right) P_{n}^{\beta+\frac{1}{p}}\right), \quad \text { since } \frac{1}{p}+\frac{1}{q}=1 .
\end{aligned}
$$


On combining equations (11) to (15), we have

$$
\left|\tilde{\tau}_{n, p}(x)-\tilde{g}(x)\right|=O\left\{\xi\left(\frac{1}{P_{n}}\right) P_{n}^{\beta+\frac{1}{p}}\right\} .
$$

The proof of the theorem is completed.

\section{Applications}

Approximation is concerned to approximate the functions by easily and simply calculated functions. The origin of approximation theory can be seen in the well-known theorem, i.e. Weirstrass Approximation theorem. This theory plays an important role in the theory of digital filters, signal and image processing. It has become an active research area for last 130 years. For designing the finite impulse response digital filter, Psarakis and Moustakides [2] gave a new method based on $L_{2}$ which improved the performance of digital filter. The following corollaries have been derived from our theorem:

Corollary 5.1. If $\beta=0$, then weighted $W\left(L^{p}, \xi(t)\right)(p \geq 1)$-class reduces to $\operatorname{Lip}(\xi(t), p)$-class and the degree of approximation of function $\tilde{g} \in$ $\operatorname{Lip}(\xi(t), p)$-class by almost Riesz means is given by

$$
\left|\tilde{\tau}_{n, p}(x)-\tilde{g}(x)\right|=O\left\{\xi\left(\frac{1}{P_{n}}\right) P_{n}^{\frac{1}{p}}\right\} .
$$

Proof. The result can be obtained by putting $\beta=0$ in (7), we get

$$
\begin{aligned}
\left|\tilde{\tau}_{n, p}(x)-\tilde{g}(x)\right| & =\left(\int_{0}^{2 \pi}\left|\tilde{\tau}_{n, p}(x)-\tilde{g}(x)\right|^{p} d t\right)^{\frac{1}{p}} \\
& =O\left(\xi\left(\frac{1}{P_{n}}\right) P_{n}^{\frac{1}{p}}\right), \quad p \geq 1 .
\end{aligned}
$$

Corollary 5.2. If $\xi(t)=t^{\alpha}, 0<\alpha \leq 1$, and $\beta=0$, then $W\left(L^{p}, \xi(t)\right)$ $(p \geq 1) \equiv \operatorname{Lip}(\alpha, p)$ class and the degree of approximation of function $\tilde{g} \in$ $\operatorname{Lip}(\alpha, p)$-class by almost Riesz means is given by

$$
\left|\tilde{\tau}_{n, p}(x)-\tilde{g}(x)\right|=O\left\{\xi\left(\frac{1}{P_{n}}\right) P_{n}^{-\alpha+\frac{1}{p}}\right\} .
$$


Corollary 5.3. Taking $p \rightarrow \infty$ in Corollary 5.2, then Lip $(\alpha, p) \equiv \operatorname{Lip} \alpha$ class and the degree of approximation of function $\tilde{g} \in$ Lip $\alpha$-class by almost Riesz means is given by

$$
\left|\tilde{\tau}_{n, p}(x)-\tilde{g}(x)\right|=O\left\{P_{n}^{-\alpha}\right\} .
$$

Proof. Taking $p \rightarrow \infty$ in the result of Corollary 5.2, we get the required result.

Remark. Independent proof of above Corollary 5.2 can be obtained along the same lines of our theorem.

\section{References}

[1] A. Zygmund, Trigonometric Series, Cambridge University Press, London (1968).

[2] E.Z. Psarakis and G.V. Moustakides, An $L_{2}$-based method for the design of 1-D zero phase FIR digital filters, IEEE Trans. Circuit and Sys. I: Fund. The. and Appl., 44, No 7 (1997), 591-601.

[3] G.G. Lorentz, A contribution to the theory of divergent series, Acta. Math., 80 (1948), 167-190.

[4] G.M. Petersen, Regular Matrix Transformations, Mc. Hill Publishing Company Ltd., London (1966).

[5] H.H. Khan, On the degree of approximation to a function to weighted $W\left(L_{p}, \xi(t)\right)$-class, Aligarh Bull. Math., 3/4 (1973-1974), 83-88.

[6] H.K. Nigam and K. Sharma, Degree of approximation of a class of function by $(C, 1)(E, q)$ means of Fourier series, IAENG Int. J. Appl. Math., 4, No 2 (2011), 1-5.

[7] H.K. Nigam and K. Sharma, Degree of approximation of a function belonging to $\operatorname{Lip}(\xi(t), r)$ class by $(E 1)(C, 1)$ product means, Int. J. Pure Appl. Math., 70, No 6 (2011), 775-784.

[8] J.P. King, Almost summable sequences, Proc. Amer. Math. Soc., 17 (1966), 120-125. 
[9] K. Qureshi, On the degree of approximation of a periodic function $f$ by almost Riesz means of its conjugate series, Indian J. Pure Appl. Math., 13, No 10 (1982), 1136-1139.

[10] K. Sharma and S.S. Malik, A study on degree of approximation of a function belonging to weighted class by product summability of Fourier series, AIP Conf. Proceedings, 2142 (2019), \# 170010; doi: 10.1063/1.5122607.

[11] K. Sharma, Estimation of error of approximation in $\operatorname{Lip}(\rho(t), r)$ class by $\left(N_{q}^{p} . C_{1}\right)$ transform, IAENG Int. J. of Applied Mathematics, 50, No 2 (2020), 251-255.

[12] L. McFadden, Absolute Nörlund summability, Duke Math. J., 9 (1942), $168-207$.

[13] M.L. Mittal, B.E. Rhoades, Degree of approximation of functions in the Holder metric, Radovi Mat., 10 (2001), 61-75.

[14] M.L. Mittal and V.N. Mishra, Approximation of signals (functions) belonging to the weighted $W\left(L_{p}, \xi(t)\right)$-class by almost matrix summability method of its Fourier series, J. Math. Sci. and Eng. Appl. (IJMSEA), 2 (2008), 285-294.

[15] N.K. Sharma and R. Sinha, On the degree of approximation of a periodic function $f$ by almost Riesz means of its conjugate series, Commun. Fac. Sci. Univ. Ank. Ser. A, 41 (1992), 111-117.

[16] P.I. Sharma and K. Qureshi, On the degree of approximation of the periodic function $f$ by almost Riesz means, Ranchi University J., 11 (1980), $29-43$.

[17] R.N. Mohapatra, Functions of class $\operatorname{Lip}(\alpha, p)$ and their Taylor mean, J. Appr. Theory, 45, No 4 (1985), 363-374.

[18] S. Nanda, Some sequence spaces and almost convergence, J. Austral. Math. Soc. Ser. A, 22 (1976), 446-455.

[19] Z.U. Ahmad and M. Mursaleen, An application of Banach limits, Proc. Amer. Math. Soc., 103, No 1 (1988), 244-246. 
